\title{
Exploring the Intermodal Relationship between Taxi and Subway in Beijing, China
}

\author{
Shixiong Jiang $\mathbb{D}^{1},{ }^{1}$ Wei Guan ${ }^{(D},{ }^{1}$ Zhengbing He, ${ }^{2}$ and Liu Yang ${ }^{1}$ \\ ${ }^{1}$ MOE Key Laboratory of Urban Transportation Complex System Theory and Technology, Beijing Jiaotong University, \\ Beijing 100044, China \\ ${ }^{2}$ College of Metropolitan Transportation, Beijing University of Technology, Beijing, China
}

Correspondence should be addressed to Wei Guan; weig@bjtu.edu.cn

Received 31 December 2017; Revised 24 May 2018; Accepted 5 June 2018; Published 28 June 2018

Academic Editor: Dongjoo Park

Copyright (C) 2018 Shixiong Jiang et al. This is an open access article distributed under the Creative Commons Attribution License, which permits unrestricted use, distribution, and reproduction in any medium, provided the original work is properly cited.

\begin{abstract}
Taxi is an indispensable mode in the urban public transportation. Although many studies have explored the travel patterns of taxi trips, few have combined taxi and subway to reveal their intermodal relationship. To bridge the gap, this study utilized taxi's trajectory data to investigate its relationship with subway. Considering the multifaceted relationship between taxi and subway in operation, taxi trips are categorized into three types, namely, subway-competing, subway-extending, and subwaycomplementing taxi trips. The characteristics of each type of taxi trips reflect the specialties and their interactions with subway. The origin/destination distributions of taxi and subway trips are compared and analyzed. Furthermore, the supply and demand of taxi within the buffer zone of each subway station are analyzed to reflect the difficulty of hailing taxis. The negative binomial regression models are used to explore the relationship between taxi trips and subway ridership. The results show that there is a significantly positive correlation between taxi trips and subway ridership.
\end{abstract}

\section{Introduction}

Taxi is a flexible on-demand public transportation, which provides passengers door-to-door services without the requirement of private car ownership. In addition, taxi has the potential to satisfy the travel demand unmet by other modes of public transportation. However, the multimodal relationships between taxi and public transit, especially subway, have not attained enough attention. Understanding the relationship can assist in providing more satisfying transportation services and encouraging mode shift from automobile to subway.

A body of studies have explored the characteristics of taxi trips. Using 20 million trajectories with fine granularity collected from more than 10 thousand taxis in Beijing, the taxis' traveling displacements in urban areas were found to follow an exponential distribution [1]. Moreover, Wang et al. [2] indicated that the displacement distributions of taxi trips follow exponential laws in two displacement ranges, while the trip duration and interevent time distributions can be approximated by log-normal distributions. In addition, there are some researches conducted from different aspects. Based on a preference travel survey, it was indicated that high population density in a user's residence area is related to longer journey durations [3]. The historical data were used to predict the number of vacant taxis in the given area and period with the prior probability distributions [4] and to predict demand distributions with respect to contexts of time, weather, and location [5]. Kamga et al. [6] found that taxi supply exhibits variations due to the decisions of taxi drivers, which are driven by both ridership levels and trip characteristics.

The pattern of subway ridership is also investigated by many researchers. Lin and Shin [7] found that daily ridership is positively affected by the floor-space area of the station areas, negatively affected by the percentage of four-way intersections, and insignificantly affected by mixed land use. Regarding travel impedance, Choi et al. [8] showed that subway travel time has the most influence on subway ridership, which represents an intrinsic property of trip length distribution. In London subway, Roth et al. [9] observed that 
intraurban movement patterns are strongly heterogeneous in terms of volume, and there is a polycentric structure composed of large flows organized around a limited number of activity centers. Similarly, Xu et al. [10] found a hierarchical urban polycentric structure composed of large concentrated flows at urban activity centers in Beijing Subway. Jiang et al. [11] observed that the displacements of subway trips follow the gamma distribution. The subway station ridership can be attributed to many factors, such as land use $[8,12]$, weather [13], and access mode $[8,14]$. Jun et al. [15] found that the population and employment density, land use mix diversity, and intermodal connectivity all have a positive impact on subway ridership, but differ in their spatial ranges. Besides, the accessibility impacts the first/last mile transport connectivity to/from the major public transit lines [16]. Walking, cycling, and bus are widely adopted as access modes for the subway stations [17-19].

Due to the congestion and pollution caused by automobile, the government is promoting the modal shift from private cars to public transit. Using data from Xian, the metro transit's influences on the mobility instrument ownership [20] and private car driving [21] are examined. The crosssectional analysis showed that metro is negatively associated with auto ownership whereas it has a positive association with bike ownership [20]. In addition, moving into metro neighborhoods is negatively associated with change in driving [21]. Huang et al. [21] concluded that metro development and the design of station-area neighborhoods have the potential to reduce driving, mitigate its impact on environment, and slow the growth of traffic congestion. Besides, the combination of taxi trips and subway ridership data can provide useful information to identify the underserved areas by public transport [22]. Examining the spatial relationship of taxi trips' origins/destinations and subway stations, Wang and Ross [23] categorized taxi trips into three types: transit-competing, transit-complementing, and transit-extending ones, to explore the inner interaction. Moreover, the geographically weighted regression was implemented to find that the urban form has a large impact on urban taxi ridership [24]. Medium income level was found to reduce the number of taxi trips at particular places and the accessibility to subways was positively associated with the taxi ridership. A high correlation between public transit ridership and taxi trips was explained by the direct demand for taxi service from major transit stations [25].

Except for the urban transport, the intercity traffic is an indispensable part for large cities. Air transport provides services in fixed terminals that require other modes, such as subway, taxi, and private car, to access them. The choice of access modes is influenced by many factors, including access time, access distance, demographic, and cost [26-28]. The quality of access mode has a significant impact on the service level of air travels, and taxi is an important mode to access airports [29]. The emerging taxi Global Positioning System (GPS) trip datasets provide the opportunity to extract travel patterns for a particular region [30]. The airport travel modal share for taxis ranges between $6 \%$ and $35 \%$ for major airports in the US, the UK, and Japan [29-31]. Spatial variables were

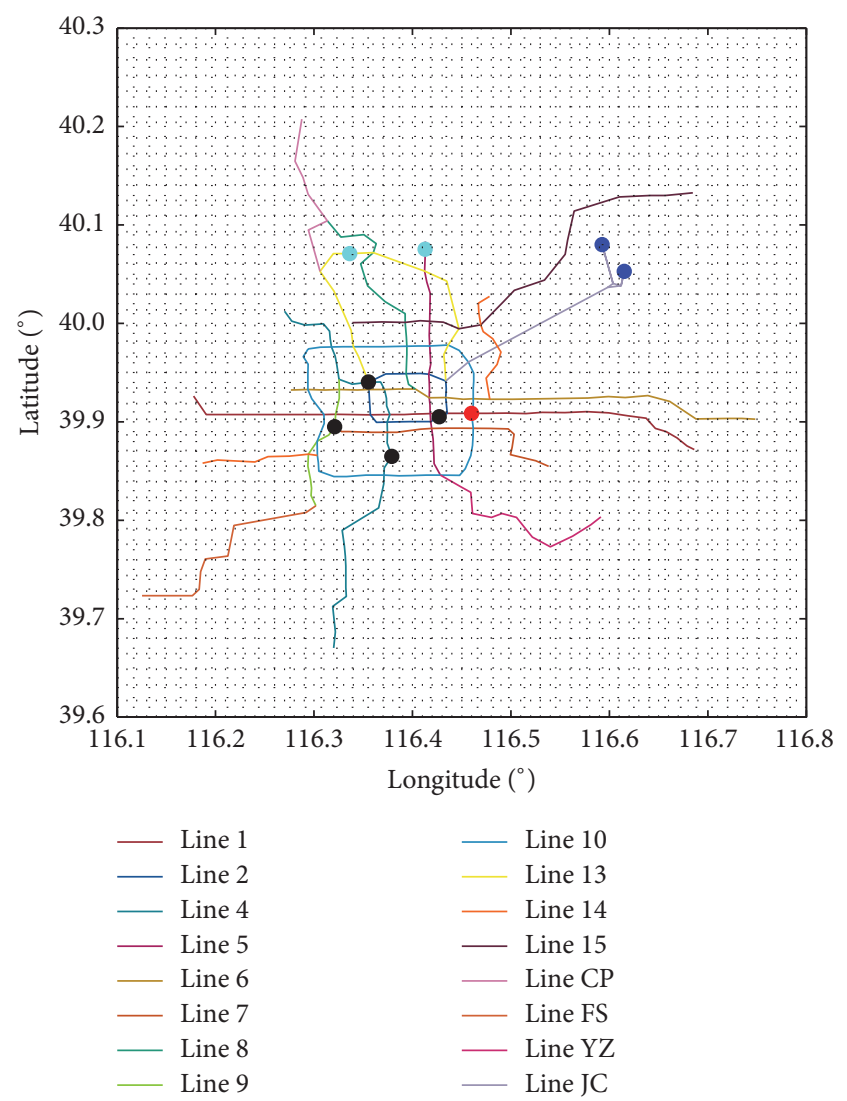

FIGURE 1: Beijing Subway map and some important points of interest. The blue point denotes the airport terminal, the black point is the railway station, the red point denotes the center business district, and the cyan point is the large residential area.

found to have the highest impact on the taxi drivers' airport pick-up decisions, followed by temporal and environmental variables [30]. In general, passengers will accept longer travel time and distance for the access travel than those for the egress travel [32].

Although many studies have investigated the travel patterns of different modes, the intermodal relationship has been rarely considered. To fill the gap, this paper aims to explore the relationship between taxi and subway. According to its relationship with subway, the taxi trips are categorized as subway-competing, subway-extending, and subwaycomplementing taxi trips. Besides, the arrival and departure taxi trips within the buffer zone of subway stations are analyzed, which are compared with the subway ridership. Furthermore, the negative binomial regression models are utilized to explore the relationship between taxi trips and subway ridership.

\section{Study Area and Data Description}

The study area is the urban part of Beijing, China (see Figure 1). There were about 67.5 thousand taxis in Beijing, which completed 0.67 billion trips (7.20\% of all trips) in 2014 [33]. As for the subway system, there were 328 stations and 
527 tracks in operation, with an average daily ridership of 9.28 million in 2014 [33].

To explore the relationship between taxi and subway, four datasets are collected, including the taxi GPS data, the subway ridership, the locations of subway stations and airports, and the points of interest (POIs) dataset. The GPS data generated by more than 46,000 taxis $[34,35]$ during January 12 th-18th, 2015, were attained [33], which contained more than 2.9 million taxi trips. The interval of the GPS data is at around 1 minute. Each record contains the location (longitude and latitude), instantaneous velocity, operational status, and so on. The objective time period was chosen since there were no major holidays or unusual weather events. A ridership dataset was available from the Automatic Fare Collection (AFC) system in Beijing Subway during December 30th-31st, 2014. It is a $268 \times 268$ origin-destination (OD) matrix, which reflects the flows between any pair of subway stations. The two datasets were collected during the common winter days without special events, although they are not in the same time period because of their different sources. In addition, the locations (longitude and latitude) of airports (Terminal 2 (T2) and Terminal 3 (T3) in Beijing Capital International Airport and Beijing Nanyuan Airport), subway stations, and the POIs dataset are collected from a map website [36] to explore how taxi trips interact with these fixed service points.

The connection between taxi and bus is not taken into consideration in this study. There are around 1020 bus routes in Beijing by 2016. Bus stops are widely spread around the city. Taking bus stops into the classification would have classified most of taxi trips as 'bus-competing' ones. Although the majority of taxi trips could be completed by bus for the large extent of the bus system, multiple transfers and long out-ofvehicle time of bus travel make it inferior to taxi [23].

\section{Methodology}

To explore the relationship between taxi and subway, the taxi trips are categorized into three types, denoted as subwaycompeting, subway-extending, and subway-complementing taxi trips [23]. And the three types are defined as follows:

(1) Subway-competing taxi trips refer to the trips which can be replaced by taking subway and acceptable walking.

(2) Subway-extending taxi trips provide connections from/to subway stations.

(3) Subway-complementing taxi trips satisfy the travels which cannot be served by subway due to the fixed routes and operation times.

Although different subway stations own various operation times, it is assumed that 5:30-23:00 is the operation time for all subway stations to simplify the problem [37].

3.1. Identify Subway-Competing Taxi Trips. Transit-competing taxi trips are trips which can be served by the subway system [23]. Subway competes with taxi only when both origin and destination of a trip are within the catchment area of subway stations because passengers can only enter and exit the subway system in stations. The walking distance threshold is widely set as $800 \mathrm{~m}$ to access subway stations $[38,39]$. Taking the road structure into consideration, the tolerant walking displacement is set as $600 \mathrm{~m}$. In this study, a taxi trip is classified as subway-competing type when its pickup and drop-off locations have subway stations within $600 \mathrm{~m}$. Additionally, the taxi trip should happen during the subway operation time. The logical process to identify the three types of taxi trips is presented in Figure 2.

3.2. Identify Subway-Extending Taxi Trips. Transit-extending taxi trips provide connection from/to subway [23]. It requires identifying taxi trips that are most likely to connect with subway stations. For such trips, taxis would originate or end close to the entrances/exits of subway stations to transfer conveniently, while another trip end is beyond the catchment area of any subway station. With regard to the size of subway stations, the maximal displacement between the entrance/exit and the station center is set as $300 \mathrm{~m}$. Based on the specific situations, two subtypes of subway-extending taxi trips are defined. The first subtype is the absolute subwayextending taxi trips that provide service between the subway stations and outskirts without subway service. The second subtype can be partly served by subway, and the other part also needs taxis to complete. The rules to select the subtype of subway-extending taxi trips are as follows: (1) only one end of a trip has a subway station within $300 \mathrm{~m}$, and the other end of the trip does not have any subway station within $2 \mathrm{~km} \mathrm{[23];}$ (2) the trip happens during the subway's operation time.

The requirements for the second subtype of subwayextending taxi trips are different. The rules are as follows: (1) only one end of a trip has a subway station within 600 $\mathrm{m}$, and the other end of the trip does not have any subway station within $2 \mathrm{~km} \mathrm{[23];} \mathrm{(2)} \mathrm{the} \mathrm{trip} \mathrm{happens} \mathrm{during} \mathrm{the}$ subway's operation time; (3) the displacement between the assumed entering and exiting subway stations is more than $2 \mathrm{~km}$.

3.3. Identify Subway-Complementing Taxi Trips. Transitcomplementing taxi trips serve the travel demand that cannot be satisfied or connected by the subway system in space or time [23]. After excluding the previous two types of taxi trips, the remained taxi trips belong to this type.

3.4. Identify Arrival and Departure Trips around Stations. As subway provides high quality travel service, subway stations' adjacent areas receive great development, which generates a large number of travel demands. To explore the taxi's supply and demand around subway stations, the area within $2 \mathrm{~km}$ from a subway station is regarded as its buffer zone. The buffer zone's originating/ending taxi trips reflect the corresponding demand/supply. The gap between the supply and demand shows the difficulty of hailing a taxi around a subway station.

In addition, taxi trips related to T3 are identified. In general, most taxis enter T3 to pick up and drop off passengers. With regard to its size, the threshold is set as $400 \mathrm{~m}$ to identify the related trips. If the pick-up point of a taxi trip is within the catchment area of T3, the taxi trip would be marked as 
TABLE 1: Summary of descriptive statistics of variables adopted in the negative binomial regression models.

\begin{tabular}{lcccc}
\hline Variable & Mean & SD & Min & 1731 \\
Subway ridership & 76459.5 & 65428.9 & 0 & 381867 \\
Automobile services & 44.8 & 65.9 & 5 & 475 \\
Life facilities & 188.4 & 124.8 & 2 & 655 \\
Recreation facilities & 53.5 & 35.1 & 1 & 213 \\
Medical facilities & 48.4 & 30.7 & 1 & 153 \\
Accommodations & 34.2 & 27.3 & 0 & 237 \\
Scenic spots & 14.3 & 27.2 & 2 & 378 \\
Government agencies & 87.6 & 68.9 & 11 & 367 \\
Transport hubs & 121.1 & 70.1 & 0 & 311 \\
Finance facilities & 55.7 & 46.2 & 73.7 & 1 \\
Companies & 88.1 & 702 \\
\hline
\end{tabular}

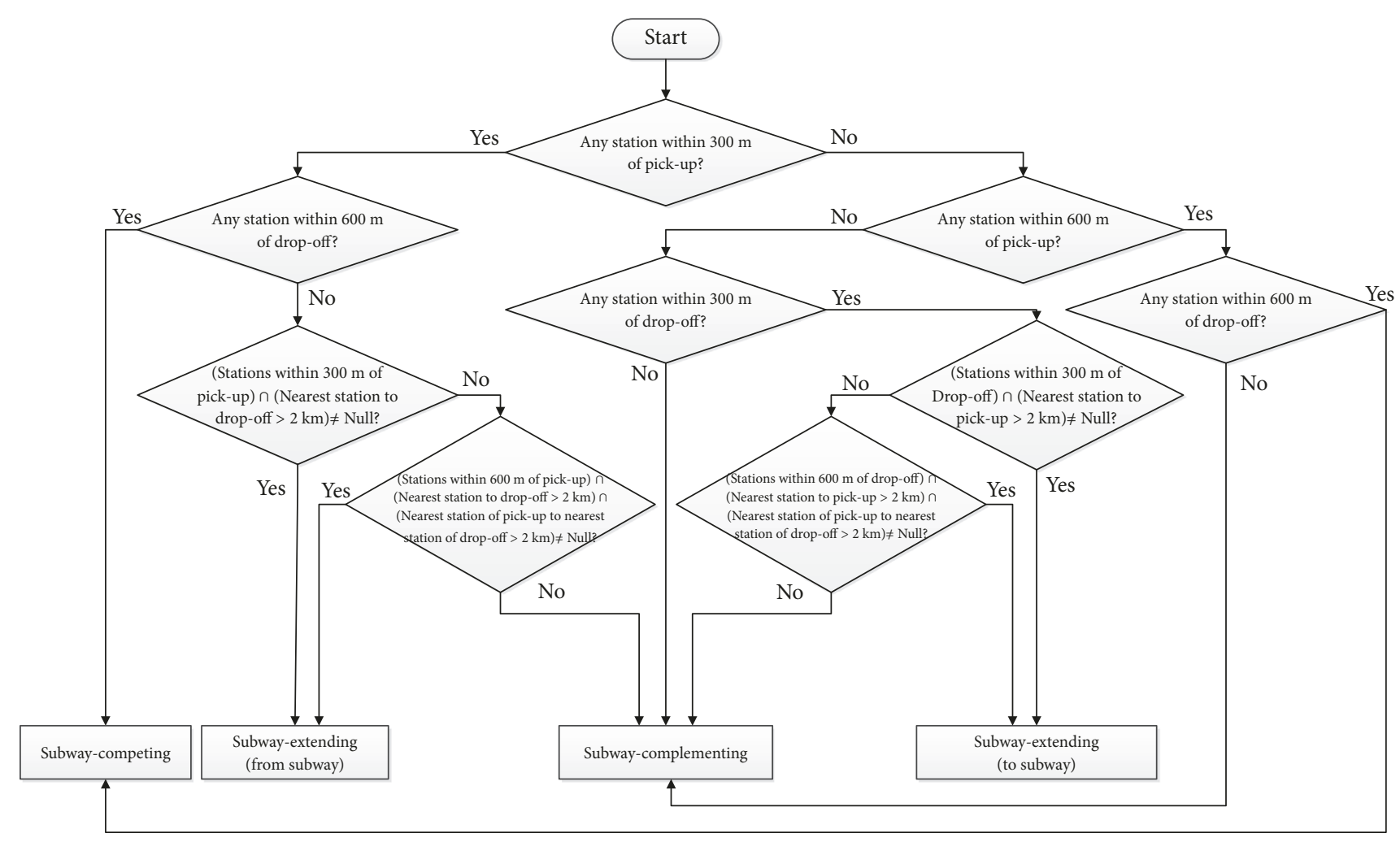

FIGURE 2: Logical flow chart to select subway-competing, subway-extending, and subway-complementing taxi trips.

a trip from T3. Similarly, a taxi trip that drops off within the catchment area of T3 is marked as a trip to T3.

3.5. Negative Binomial Regression Model. To investigate the effect of contributing factors on the taxi ridership and to assess the effect of subway ridership in particular, the negative binomial regression models were fitted [40]. The taxi trips in the subway station buffers, subway-competing taxi trips, and subway-extending taxi trips are explored, respectively. The independent variables used in this study include subway ridership, automobile services, life facilities, recreation facilities, accommodations, scenic spots, government agencies, transport hubs, finance facilities, and companies (see Table 1).

\section{Results}

4.1. Characteristics of Taxi Trips. Among the 2,918,143 taxi trips, there are $19.32 \%$ for subway-competing, $4.58 \%$ for subway-extending, and $76.10 \%$ for subway-complementing taxi trips (see Table 2), which indicates that many travels cannot be satisfied by the subway system. The responding proportions are quite different from those in New York City [23], in which the transit-competing taxi trips took up $58.50 \%$ of all taxi trips.

4.1.1. Travel Distance. The travel distance distributions of the three types of taxi trips are different (see Figure 3). Overall, the average travel distance is $9.59 \mathrm{~km}$ for all taxi trips, and 
TABLE 2: Characteristics of three types of taxi trips.

\begin{tabular}{lccc}
\hline Characteristic & Subway-competing & Subway-extending & Subway-complementing \\
\hline Average travel distance $(\mathrm{km})$ & 9.61 & 14.26 & 9.30 \\
Average travel time $(\mathrm{min})$ & 22.23 & 26.99 & 20.33 \\
Count & 563,851 & 133,546 & $2,220,746$ \\
Percentage (\%) & 19.32 & 4.58 & 76.10 \\
\hline
\end{tabular}

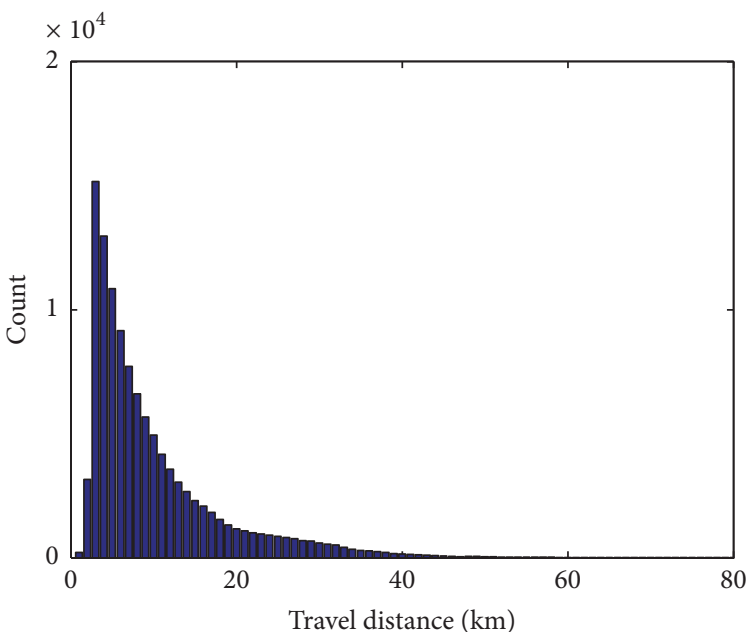

(a)

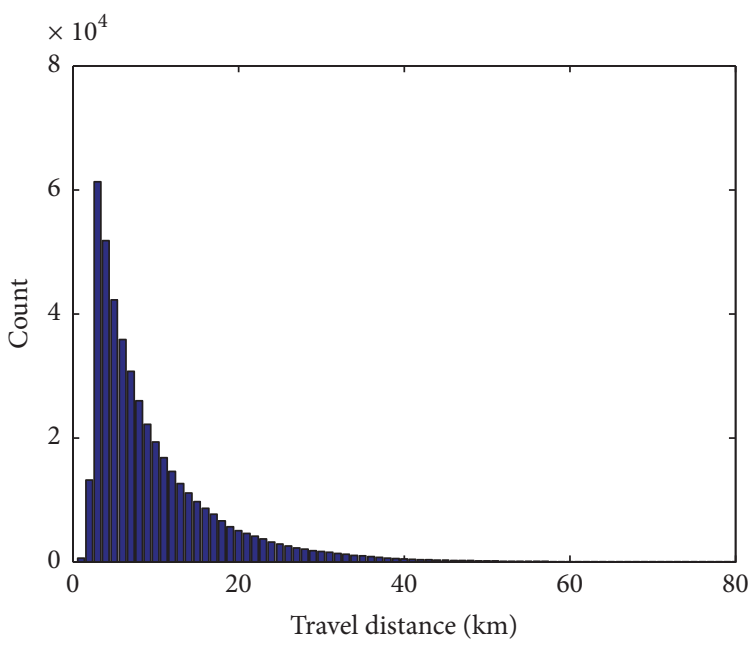

(c)

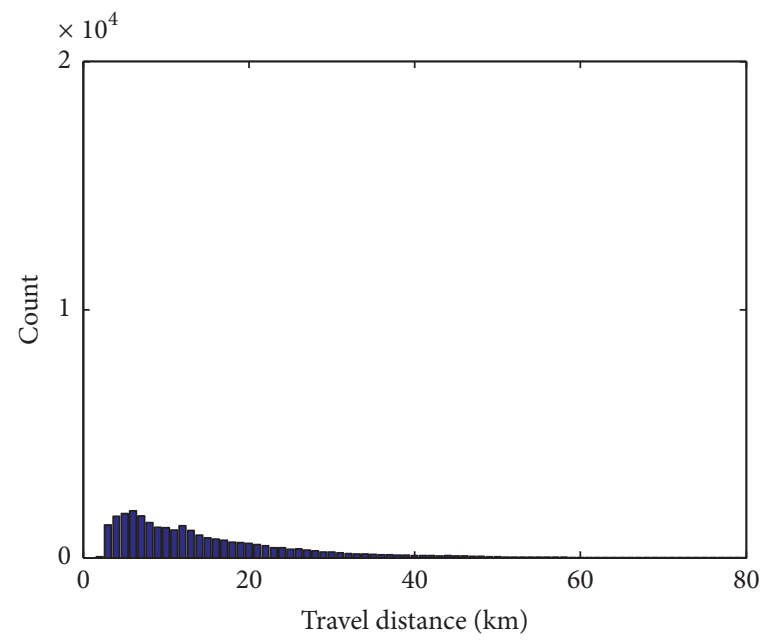

(b)

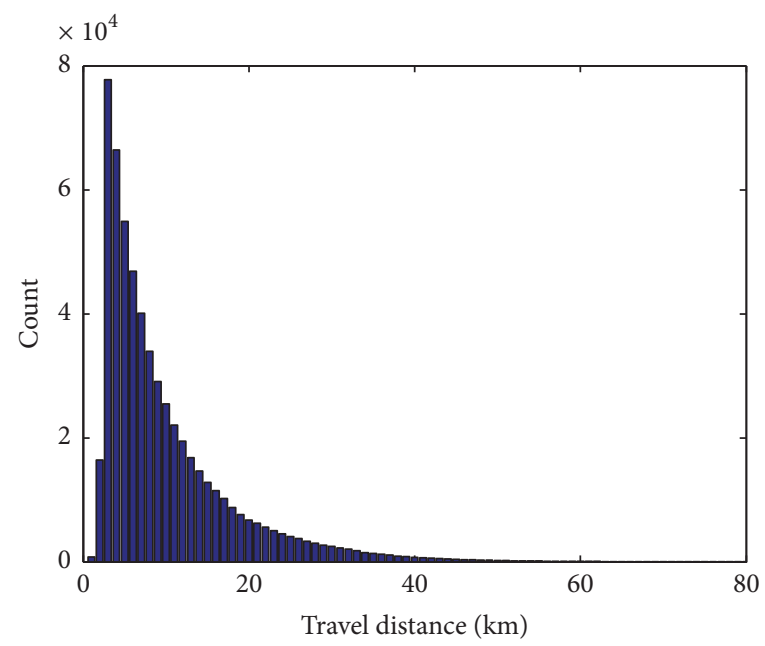

(d)

Figure 3: The travel distance distributions of taxi trips. (a) Subway-competing taxi trips. (b) Subway-extending taxi trips. (c) Subwaycomplementing taxi trips. (d) All taxi trips.

$89.6 \%$ of taxi trips are less than $20 \mathrm{~km}$. For the subwaycompeting and subway-complementing taxi trips, $67.8 \%$ and $68.3 \%$ of trips are within $10 \mathrm{~km}$, respectively. Besides, a $20-\mathrm{km}$ travel distance covers $88.9 \%$ of subway-competing taxi trips and $90.5 \%$ of subway-complementing ones. However, the travel distance is apparently longer for the subway-extending taxi trips. There are only $45.9 \%$ of subway-extending taxi trips within $10 \mathrm{~km}$ and $77.8 \%$ of those within $20 \mathrm{~km}$, which implies that they are more likely to relate to peripheries.
4.1.2. Spatial Distribution. The pick-ups and drop-offs spatial distributions of the three types of taxi trips are shown in Figures 4, 5, and 6, respectively. The numbers of pick-ups and drop-offs are mapped at the grid cell level $\left(0.01^{\circ}\right.$ latitude $\times 0.01^{\circ}$ longitude $)$. Subway-competing taxi trips are concentrated on the urban area, while subwayextending taxi trips tend to spread in the peripheries. Subway-complementing taxi trips are widely spread in spatial scale. 


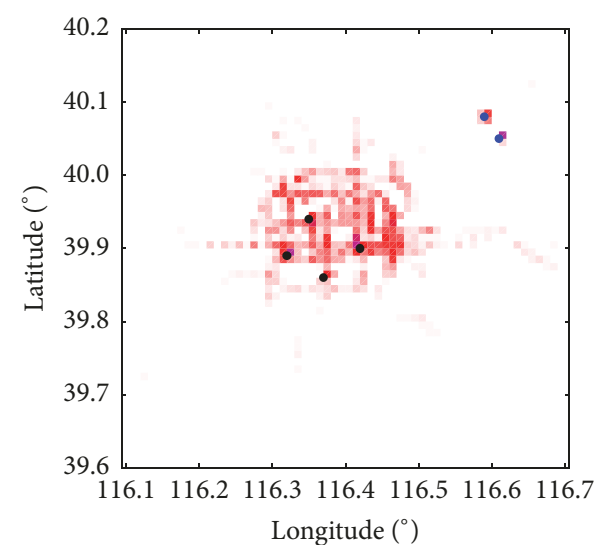

(a)

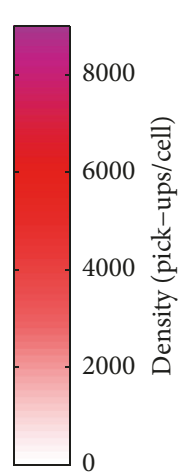

Figure 4: The spatial distributions of subway-competing taxi trips. (a) Pick-ups. (b) Drop-offs.

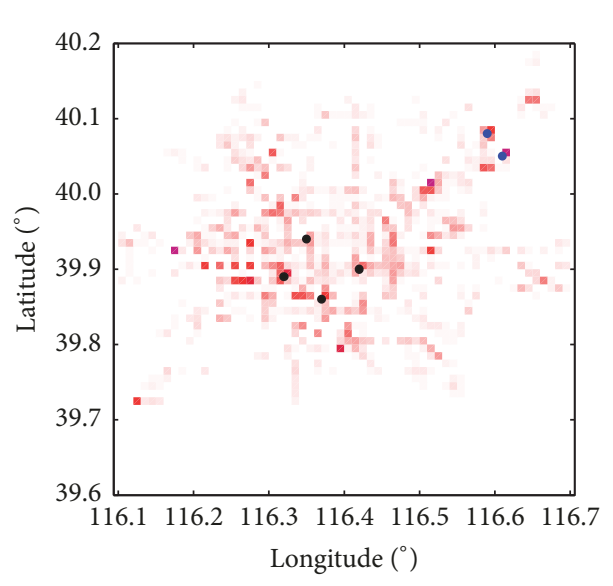

(a)

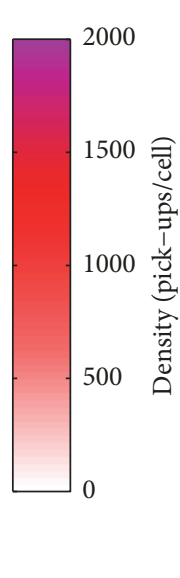

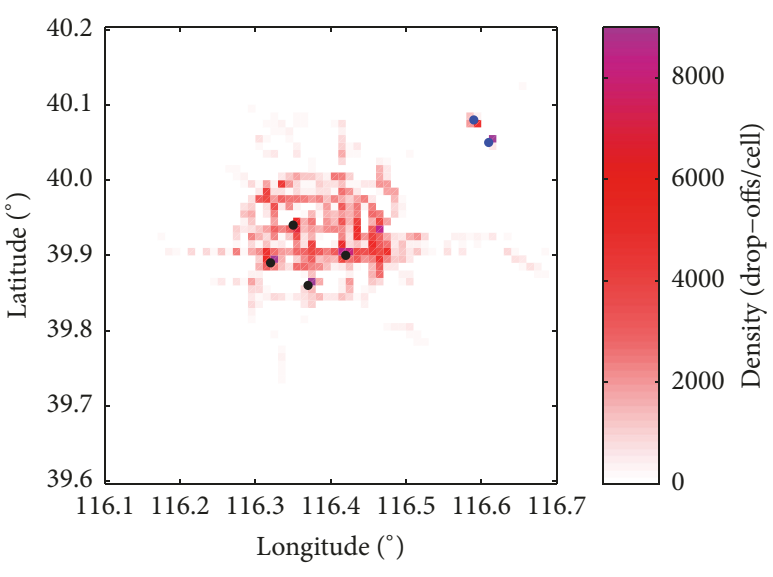

(b)

FIgUre 5: The spatial distributions of subway-extending taxi trips. (a) Pick-ups. (b) Drop-offs.

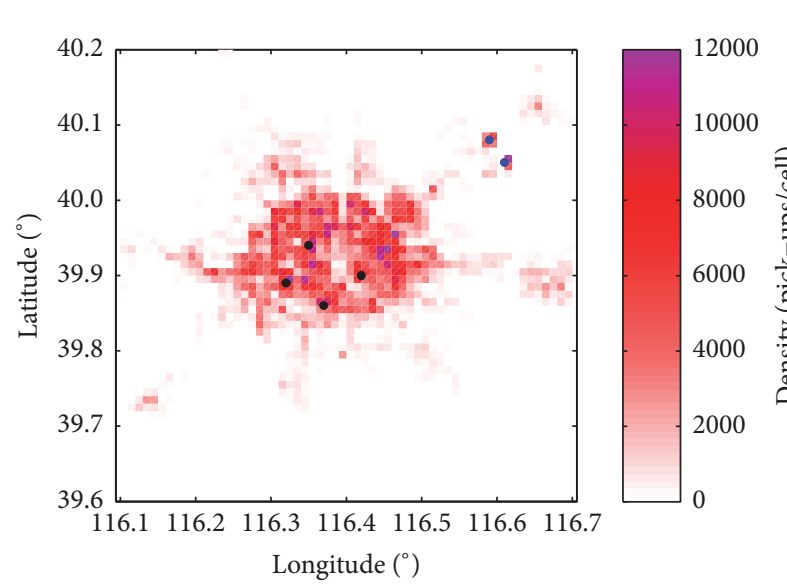

(a)

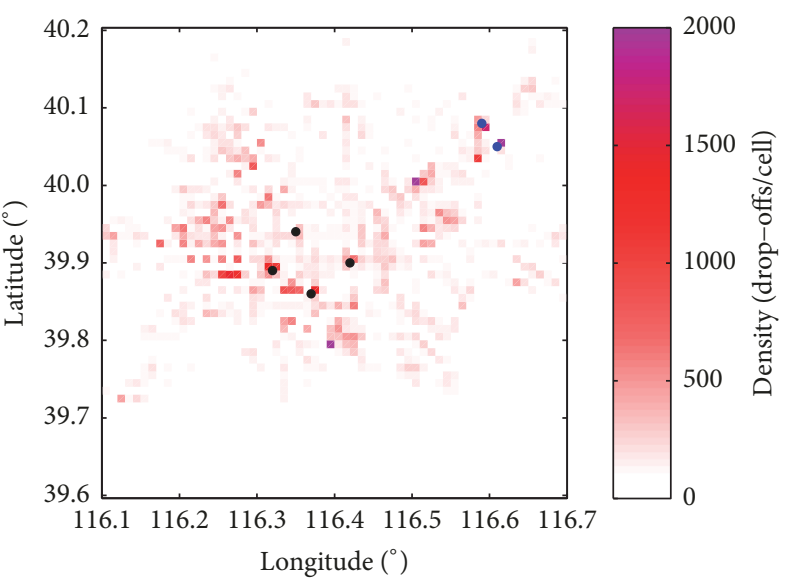

(b) 


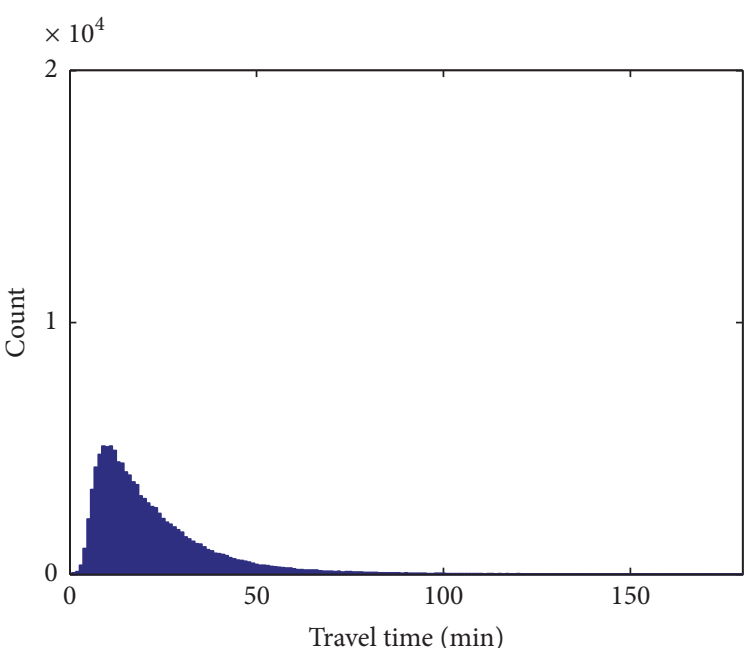

(a)

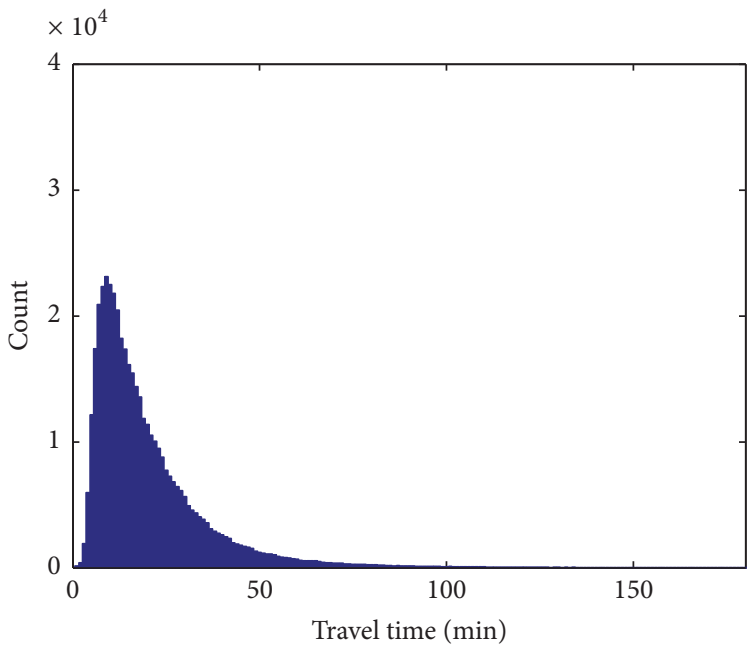

(c)

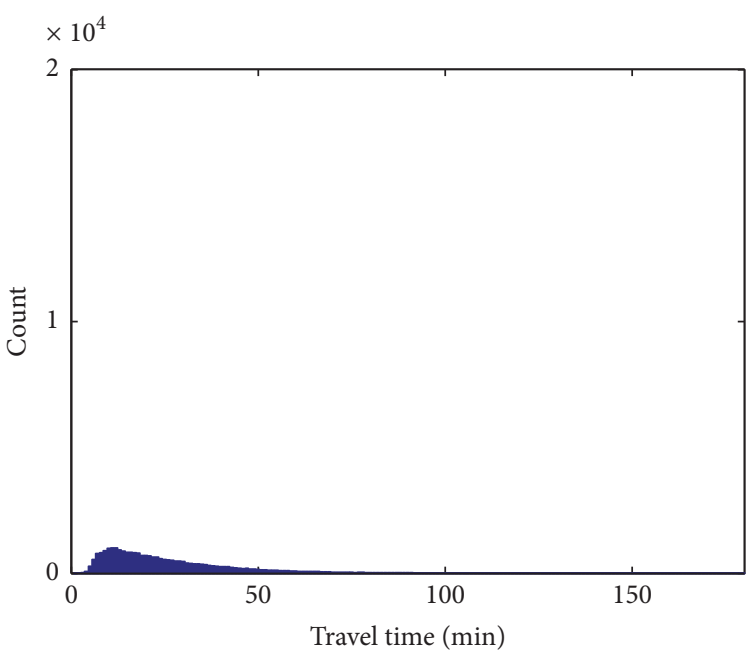

(b)

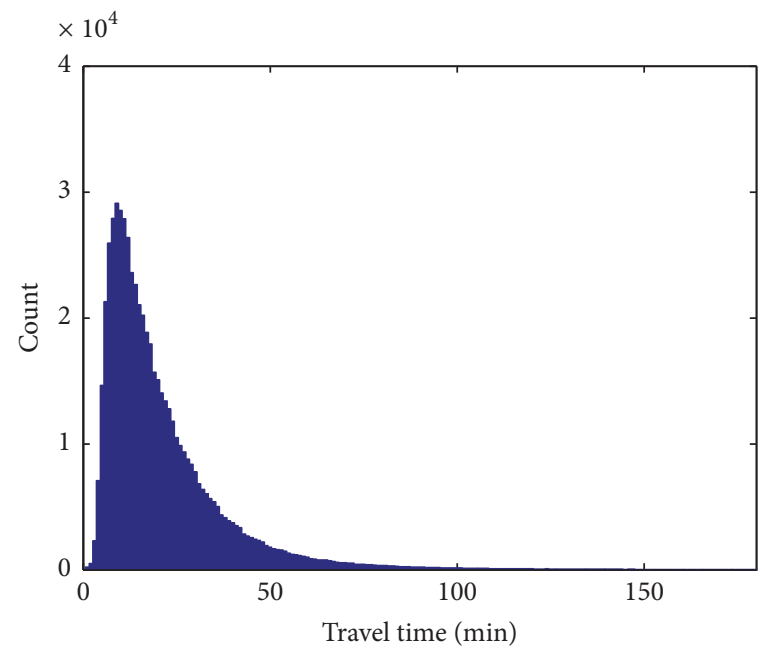

(d)

FIgure 7: The travel time distributions of taxi trips. (a) Subway-competing taxi trips. (b) Subway-extending taxi trips. (c) Subwaycomplementing taxi trips. (d) All taxi trips.

4.1.3. Travel Time. The travel time is also an important indicator for both passengers and taxi drivers. The distributions of travel times are plotted in Figure 7. Overall, the travel time averages at $21.00 \mathrm{~min}$ and $96.7 \%$ of taxi trips are less than $60 \mathrm{~min}$. In detail, there are $78.7 \%, 69.3 \%$, and $82.6 \%$ of trips within $30 \mathrm{~min}$ for subway-competing, subway-expending, and subway-complementing taxi trips, respectively. However, the three types have similar percentages around $95 \%$ within $60 \mathrm{~min}$.

Combining the travel distance and travel time, the average speed can be computed. The average speed of subwayextending taxi trips reaches $33.49 \mathrm{~km} / \mathrm{h}$, which is significantly higher than the other two types. The average speeds are only $26.86 \mathrm{~km} / \mathrm{h}$ and $29.11 \mathrm{~km} / \mathrm{h}$ for subway-competing and subway-complementing taxi trips, respectively.

4.1.4. Departure Time. The departure time distributions of taxi trips are presented in Figure 8. Generally, over 91.0\% of taxi trips happen from 8:00 to 24:00, with two peaks at around 10:00 and 14:00, and the bottom is at 4:00 in the early morning. Because subway does not operate all day, subwaycompeting and subway-extending taxi trips are limited in the operation time. The subway-competing taxi trips reach the peak at about 13:00.

The average travel distance and average travel time by time of day are plotted in Figure 9. The three types of taxi trips show a similar trend, while the average travel distance of subway-extending taxi trips is significantly higher than other two types. The average distance has a peak at around 6:00 and a bottom at the midnight. However, the average travel time does not show higher value. The average travel distance is normal in the morning and evening peak hours, while the average travel time shows apparent peaks during peak hours, which might be attributed to the traffic congestion. 


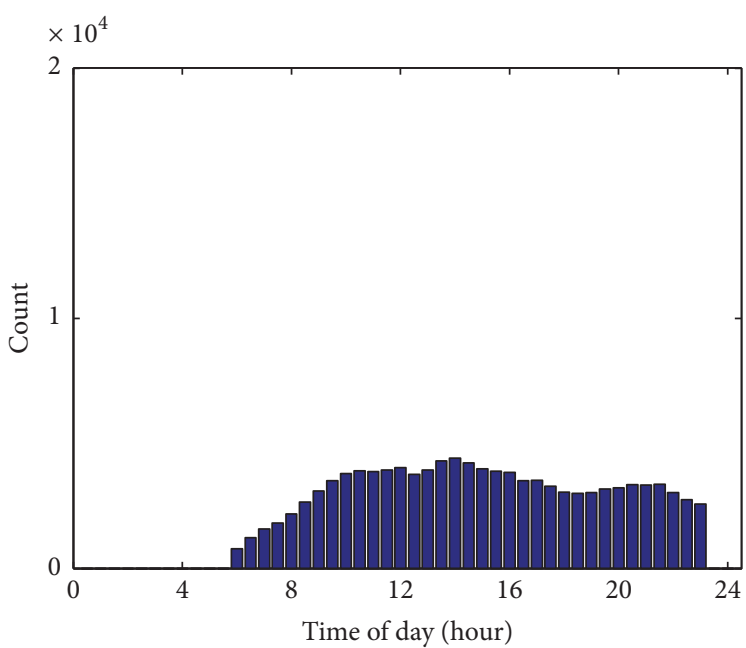

(a)

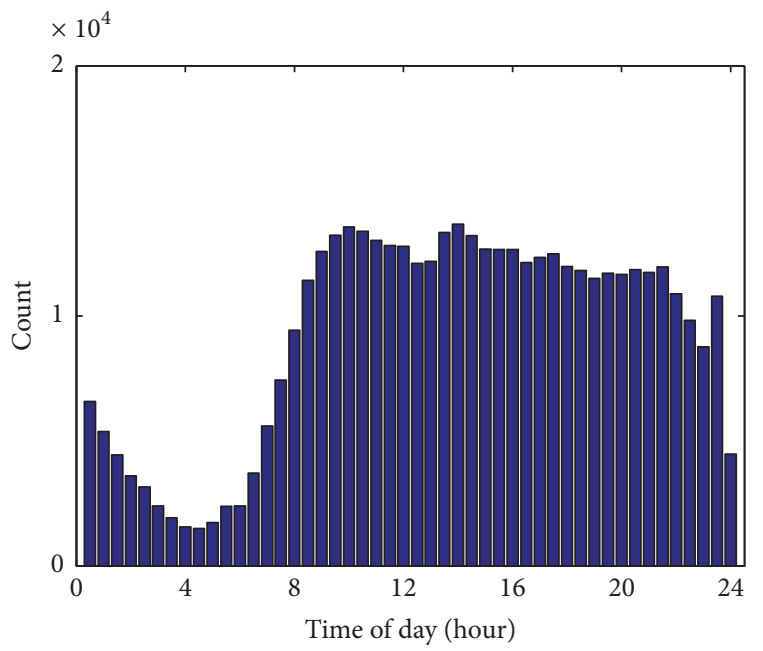

(c)

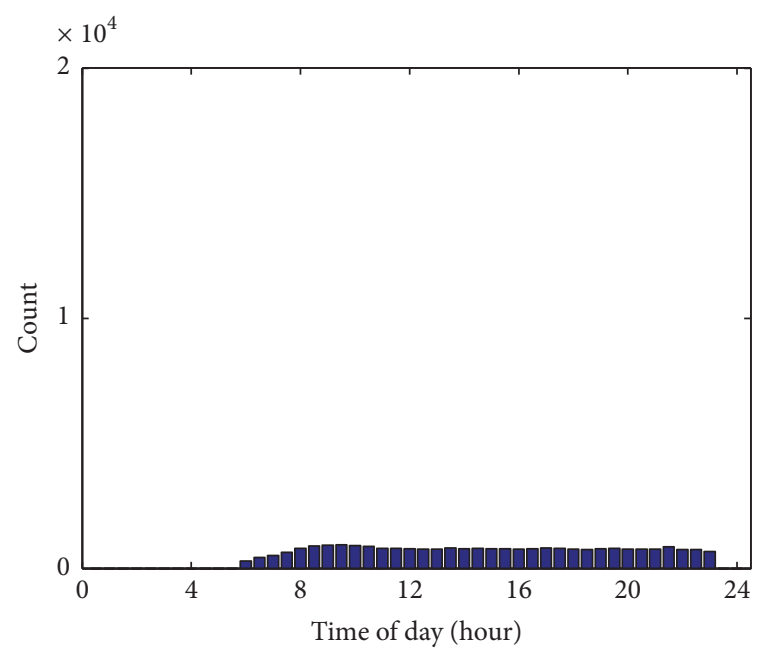

(b)

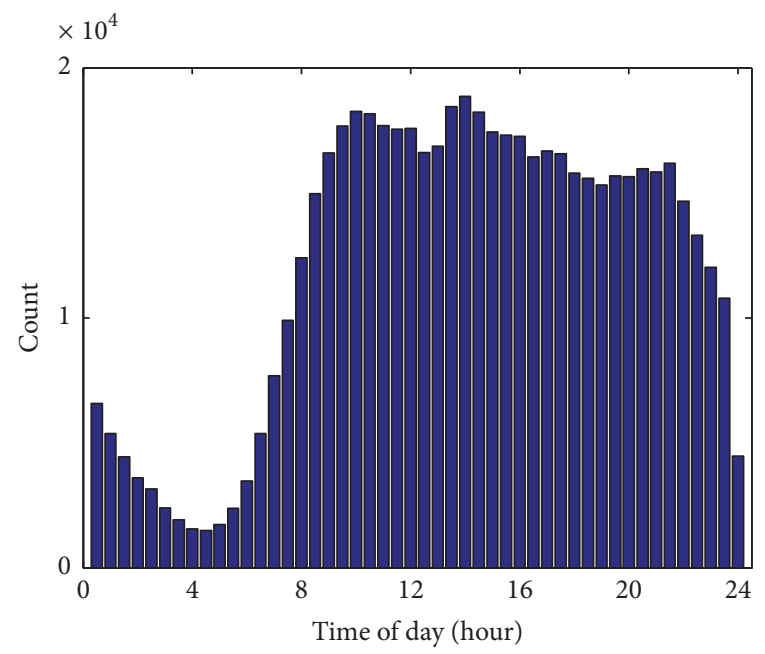

(d)

Figure 8: The departure time distributions of taxi trips. (a) Subway-competing taxi trips. (b) Subway-extending taxi trips. (c) Subwaycomplementing taxi trips. (d) All taxi trips.

\subsection{Spatial Distribution of Arrival and Departure Trips}

4.2.1. Subway Ridership Spatial Distribution. The spatial distributions of subway ridership are presented in Figure 10. There are some stations with apparently higher ridership, with most being in the downtown area and some in the peripheral areas. According to Figure 10, the arrival and departure trips show a similar spatial distribution, with the Spearman correlation of $0.990(p<0.01)$, because the subway trips are more likely to be commuting ones, which would be round trips in a day. To explore the difference between the arrival and departure ridership, the gap between arrival and departure is plotted in Figure 11. Most subway stations with a large gap (more arrival than departure) are near the railway stations and airports. On the contrary, many subway stations in residential areas have less arrivals than departures.

4.2.2. Spatial Distribution of Taxi Trips in Buffer Zones of Subway Stations. The arrival and departure trips of taxi in the buffer zones are plotted in Figure 12. When a taxi arrives its destination, it will be vacant, which means the supply of taxi. Similarly, the originating taxi trip implies the demand for taxi. Generally, the arrival and departure of taxi trips also show similar spatial distribution, with the Spearman correlation of $0.988(p<0.01)$. The high pick-up and drop-off zones are located in the urban areas, and they are more dispersed than the subway trip distribution. Besides, there are also some exceptions. For example, T2 and T3 also show a high value of pick-ups and drop-offs.

The buffer zones of subway stations have developed rapidly and there are a large amount of travel demands. The gap between supply and demand of each buffer zone can represent the difficulty of hailing taxis (see Figure 13). The over-demand areas are mainly located in the north central part of the city. On the other hand, there are also some oversupply areas spreading around the suburbs. Particularly, the buffer zones of airports and railway stations are the most over-supply areas. 


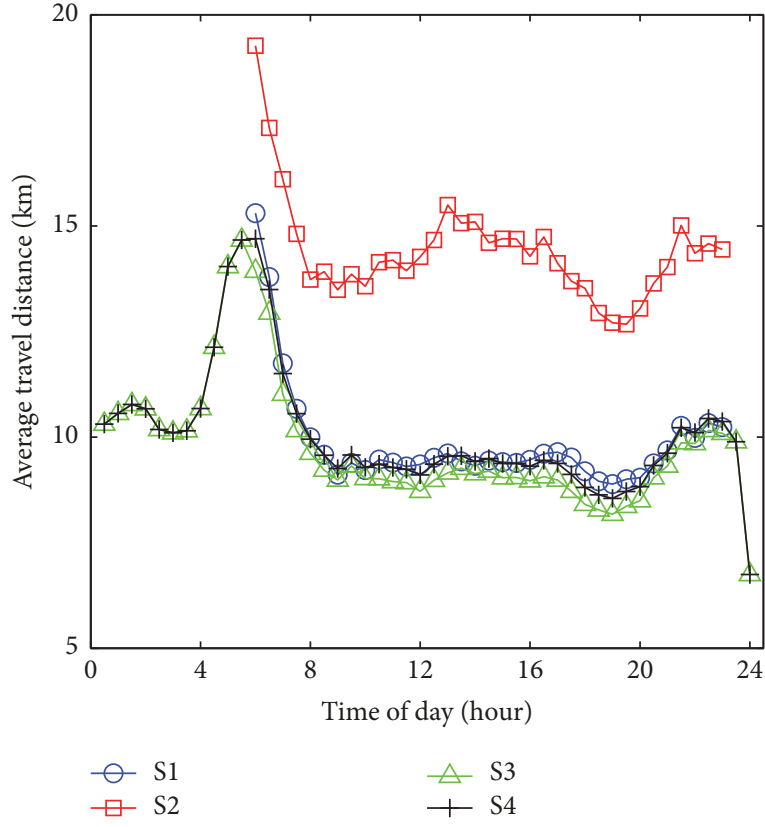

(a)

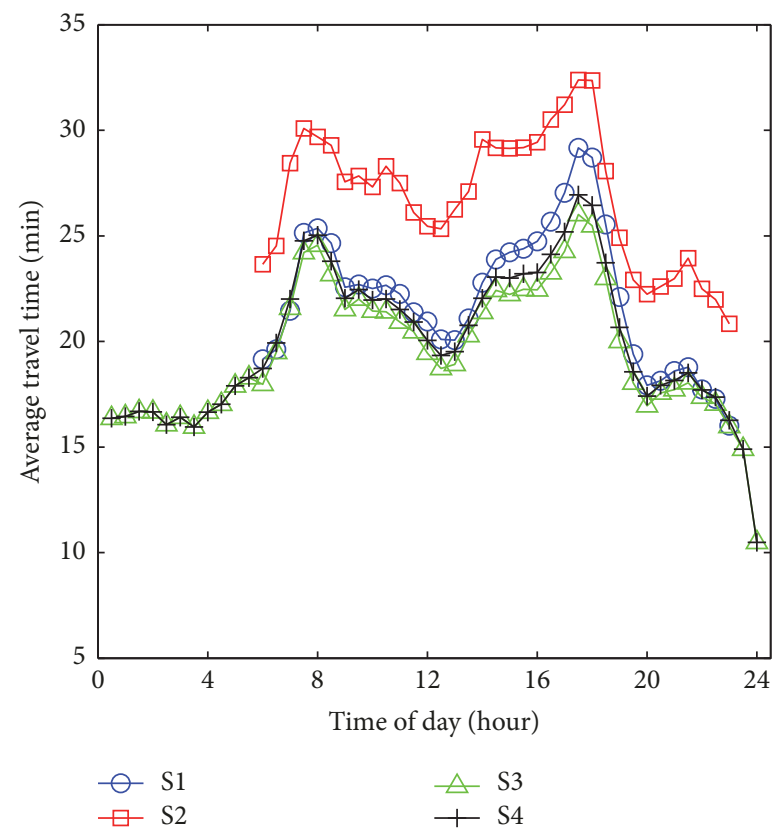

(b)

FIGURE 9: The average travel distance and average travel time by time of day. (a) The average travel distance. (b) The average travel time. (S1: subway-competing taxi trips; S2: subway-extending taxi trips; S3: subway-complementing taxi trips; S4: all taxi trips.)

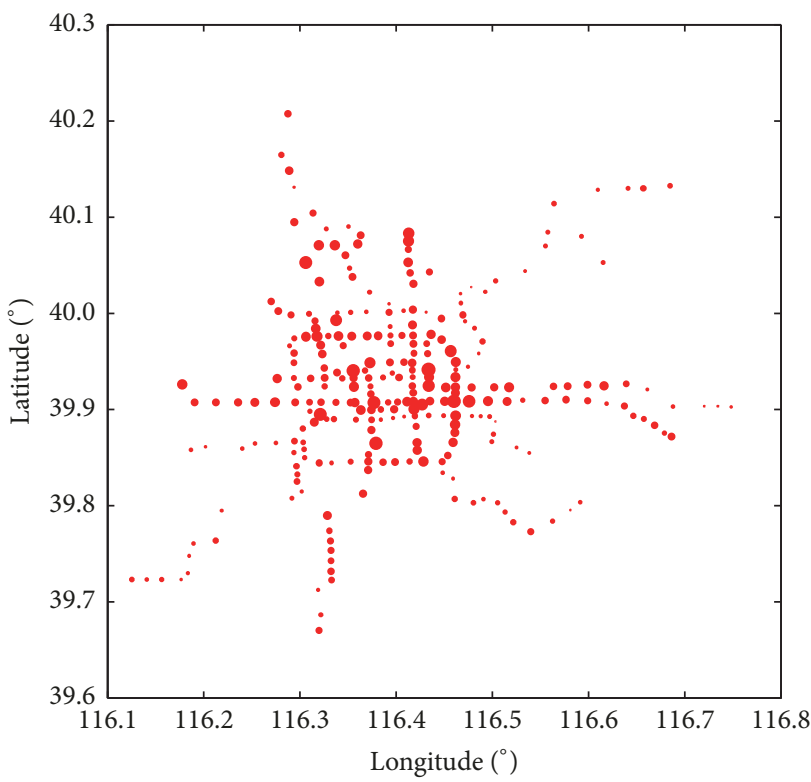

(a)

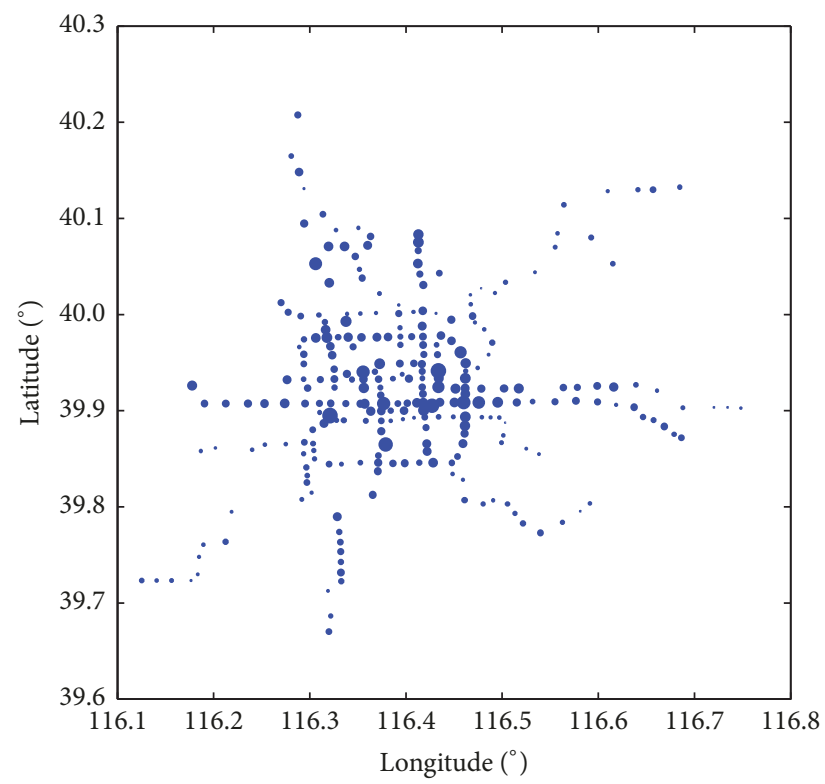

(b)

Figure 10: The arrival and departure of subway trips. (a) Arrival of subway trips. (b) Departure of subway trips.

According to Figures 10 and 12, the ridership spatial distributions are different between subway and taxi. The subway system has some stations with extremely high ridership, while the taxi trips are more dispersed. It is interesting to find that there are much more arrival trips than departure ones by subway and taxi in both railway stations and airports. Besides, it is necessary to consider the ridership patterns of subway and taxi in time of day.
4.2.3. Subway and Taxi Trips in T3. For large cities, there are a great quantity of travelers adopting subway and taxi to access airports. Understanding the travel patterns of these trips can help to provide more appropriate access/egress services. Taxi trips to airports and those from airports are identified according to the pick-up and drop-off locations. The arrival and departure of taxi and subway trips related to T3 are presented in Figure 14. There is an apparent peak at 7:00 in the 
TABLE 3: Characteristics of taxi trips connecting to T3.

\begin{tabular}{lcc}
\hline Characteristic & From T3 & To T3 \\
\hline Average travel distance $(\mathrm{km})$ & 29.15 & 26.83 \\
Average travel time $(\mathrm{min})$ & 41.40 & 35.01 \\
Count & 22,903 & 25,706 \\
\hline
\end{tabular}

TABLE 4: Results of the negative binomial regression models for subway-competing taxi trips and taxi trips in buffer zones of subway stations.

\begin{tabular}{|c|c|c|c|c|c|c|}
\hline \multirow[b]{2}{*}{ Parameter } & \multicolumn{3}{|c|}{ Model 1: subway-competing taxi trips } & \multicolumn{3}{|c|}{ Model 2: taxi trips in buffer zones } \\
\hline & Coefficient & Standard error & $\mathrm{p}$-value & Coefficient & Standard error & p-value \\
\hline Intercept & $7.02^{* *}$ & 0.185 & 0.000 & $8.47^{* *}$ & 0.135 & 0.000 \\
\hline Subway ridership & $5.39 \mathrm{e}-06^{* *}$ & $1.04 \mathrm{e}-06$ & 0.000 & $2.84 \mathrm{e}-06^{* *}$ & $6.54 \mathrm{e}-07$ & 0.000 \\
\hline Automobile services & $-4.17 e-03^{* *}$ & $8.77 e-04$ & 0.000 & $-1.37 \mathrm{e}-03^{*}$ & $5.49 e-04$ & 0.013 \\
\hline Life facilities & $-6.55 e-03^{* *}$ & $9.43 e-04$ & 0.000 & $-3.47 \mathrm{e}-03^{* *}$ & $6.28 \mathrm{e}-04$ & 0.000 \\
\hline Recreation facilities & $7.58 \mathrm{e}-03^{* *}$ & $2.23 e-03$ & 0.001 & $5.83 e-03^{* *}$ & $1.55 \mathrm{e}-03$ & 0.000 \\
\hline Accommodations & $7.28 \mathrm{e}-03^{*}$ & $2.99 \mathrm{e}-03$ & 0.015 & & & \\
\hline Medical facilities & & & & $5.96 \mathrm{e}-03^{* *}$ & $2.03 \mathrm{e}-03$ & 0.003 \\
\hline Government agencies & $4.80 \mathrm{e}-03^{* *}$ & $1.40 \mathrm{e}-03$ & 0.001 & $2.53 \mathrm{e}-03^{* *}$ & $9.21 \mathrm{e}-04$ & 0.006 \\
\hline Transport hubs & $3.20 \mathrm{e}-03^{+}$ & $1.65 e-03$ & 0.052 & $4.47 \mathrm{e}-03^{* *}$ & $1.19 \mathrm{e}-03$ & 0.000 \\
\hline Finance facilities & $1.37 \mathrm{e}-03^{* *}$ & $3.21 \mathrm{e}-03$ & 0.000 & $6.01 \mathrm{e}-03^{* *}$ & $1.53 e-03$ & 0.000 \\
\hline Companies & $-2.66 \mathrm{e}-03^{*}$ & $1.31 \mathrm{e}-03$ & 0.043 & & & \\
\hline
\end{tabular}

Note. $* *: p<0.01, *: p<0.05,+: p<0.10$

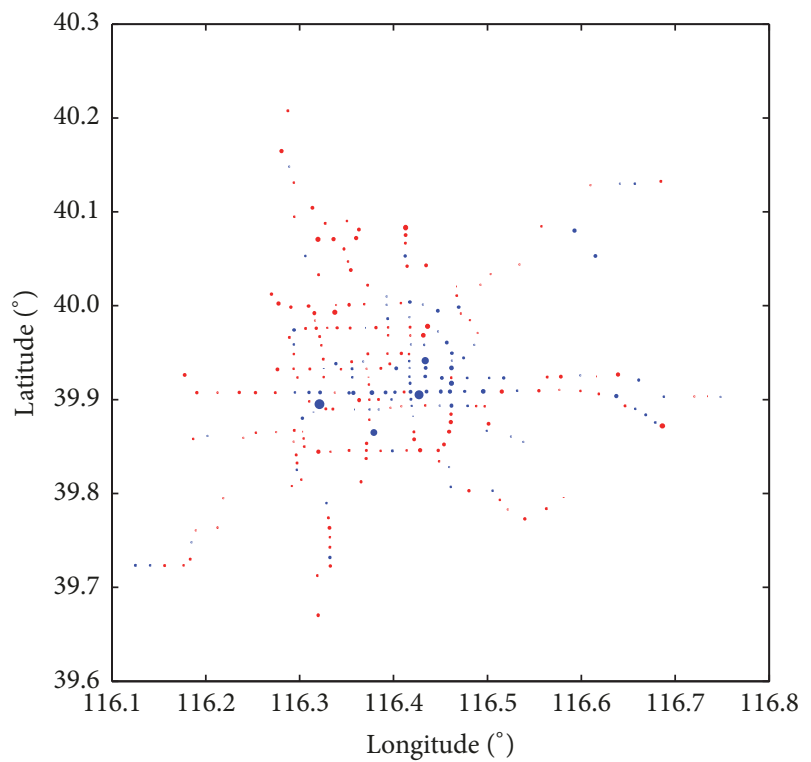

FIGURE 11: The gap between arrival and departure subway trips. The blue circles denote that the arrival trips are more than departure trips. The red circles denote that the arrival trips are less than departure trips.

morning for taxi trips to T3. There are a stable amount of taxi trips originating from airports during 10:00-24:00 and some during 0:00-2:00, while the departure trips are much lower in other time periods of a day, but for taxi trips to airports, it is few in the midnight. From 8:00 to 16:00, the number of taxi trips is stable, then it decreases slowly. As shown in Figure 14, it is unbalanced between the supply of taxi and the demand for taxi at T3. From 4:00 to 16:00, there is more supply than demand, while it is opposite in other time periods. It is found that it is easier for taxi drivers to pick up passengers at T3 after 16:00.

Comparing the taxi and subway trips at T3, the taxi and subway trips from T3 have a similar overall trend, low volumes in the morning and high volumes in the afternoon. However, the taxi trips to T3 see the peak at 6:00, while the subway trips to T3 reach the peak at 19:00. To conclude, the taxi and subway trips from T3 have similar patterns, while those trips to T3 have different patterns.

In all, there are more taxi trips to airports than those from T3 (see Table 3). As the airports are rather far from the downtown and there are few taxi demands in adjacent areas, about 2,800 taxis would go back to the downtown without any passenger in one day. The average travel distance and average travel time of taxi trips to T3 are slightly less than those of taxi trips from T3.

\subsection{Relationship between Taxi Trips and Subway Ridership.} The variables used in the negative binomial regression models were selected carefully to avoid collinearity, and different models are selected according to the Akaike information criterion (AIC) and log-likelihood values [22,23]. Table 4 shows the results of the negative binomial regression models for estimating the number of subway-competing taxi trips and taxi trips in the buffer zones of subway stations. The results show that subway ridership, recreation facilities, government agencies, transport hubs, and finance facilities are positively associated with taxi trips in both models. A high correlation between subway ridership and taxi trips is consistent with the previous study [25] and can be explained by the direct 


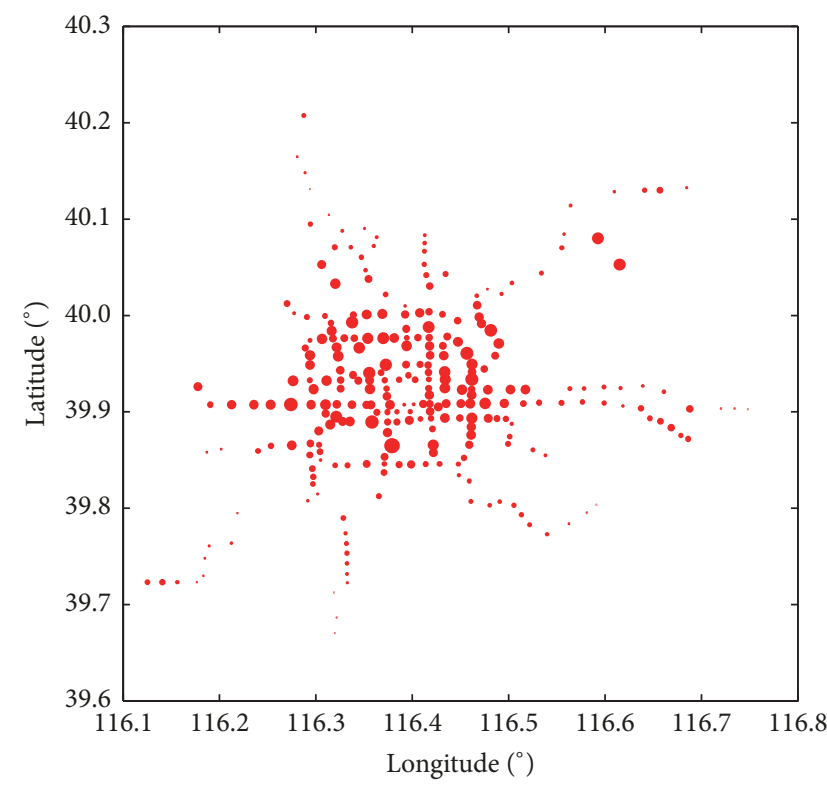

(a)

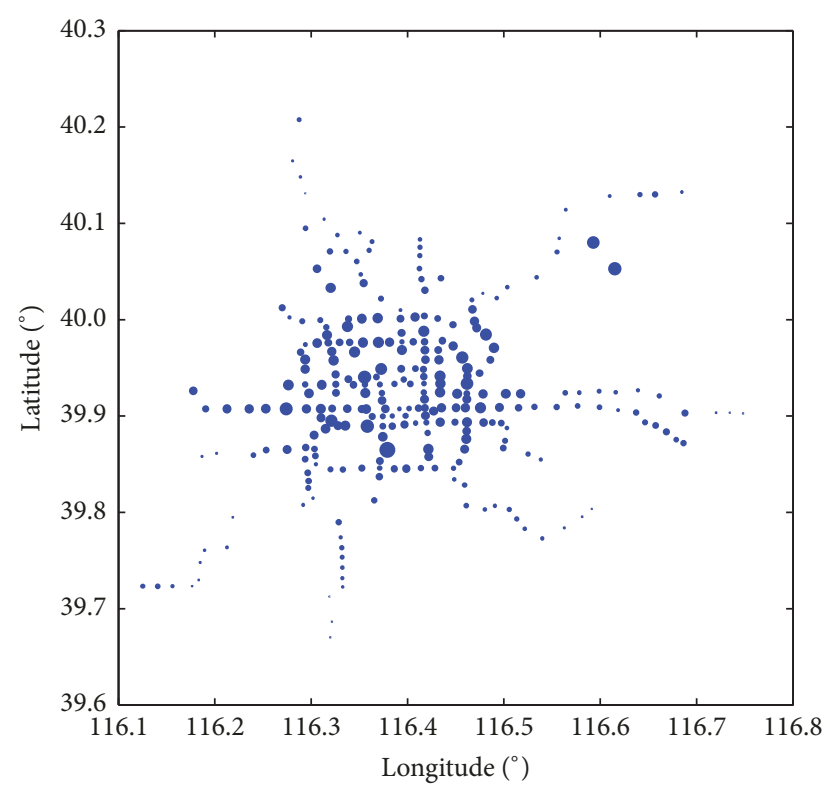

(b)

FIGURE 12: The arrival and departure of taxi trips in buffer zones of subway stations. (a) Arrival of taxi trips. (b) Departure of taxi trips.

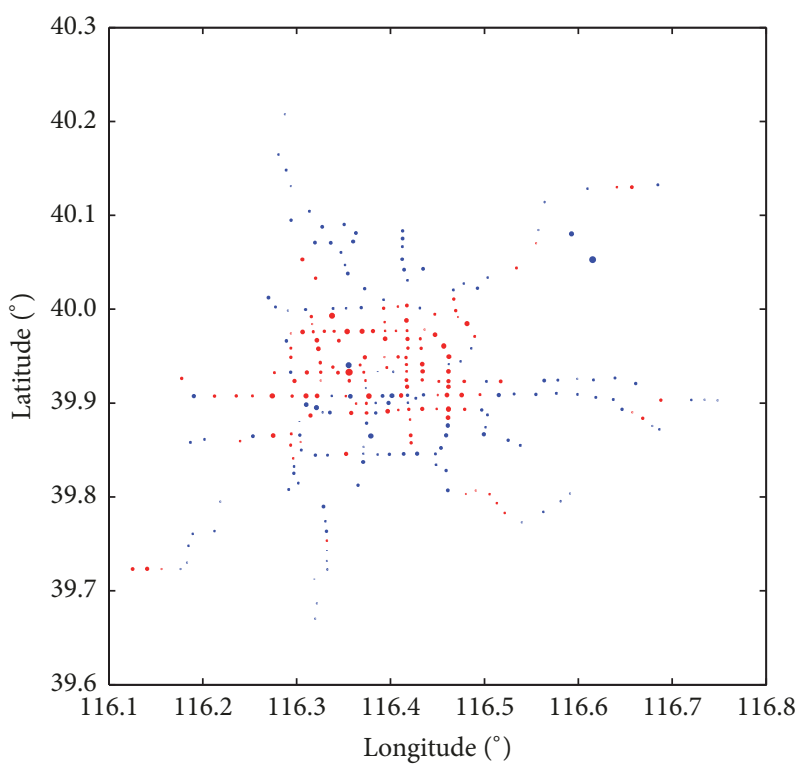

FIGURE 13: The gap between arrival and departure taxi trips in buffer zones of subway stations. The blue circles denote that the arrival trips are more than departure trips. The red circles denote that the arrival trips are less than departure trips.

demand for taxi service from subway stations $[22,25]$. The coefficients of subway ridership, recreation facilities, and government agencies are larger in model 1 than those in model 2. However, automobile services and life facilities variables have negative coefficients in model 2 and larger negative coefficients in model 1 . Besides, accommodations variable has a positive coefficient in model 1 , which is more related to visitors from other cities. It suggests that

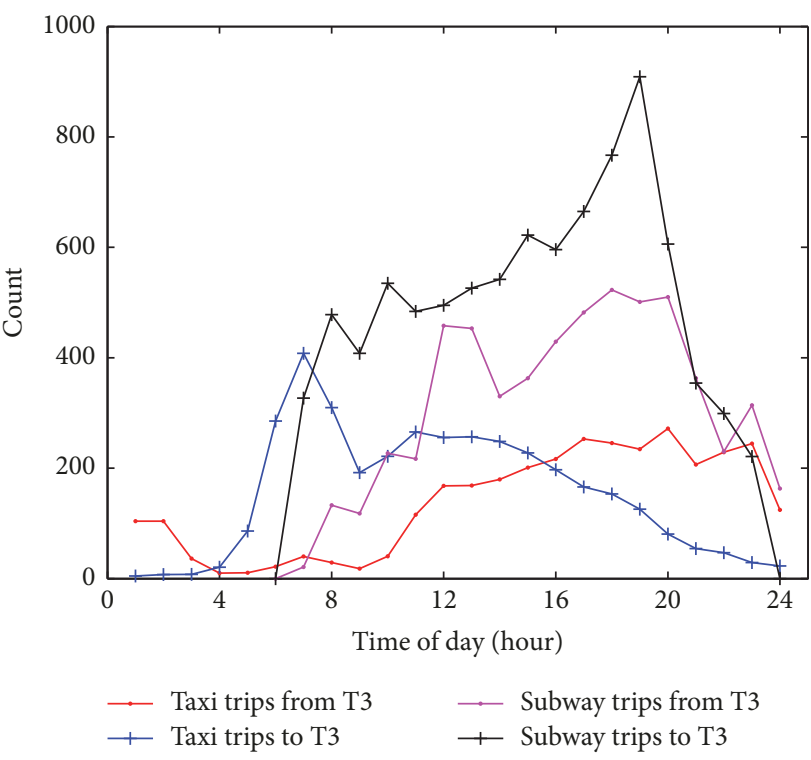

FIGURE 14: The taxi and subway trips from/to T3 by time of day.

visitors prefer taxi than subway. The companies variable has a negative coefficient in model 1 , which is likely to relate to daily commuters who tend to take subway. Furthermore, medical facilities variable is positively associated with taxi trips in station buffers in model 2, which indicates that passengers who are not well in health prefer to take a taxi to get better services.

In addition, the subway-extending taxi trips are also explored by the negative binomial regression model. Three variables associated with the subway-extending taxi trips are presented in Table 5. Subway ridership and transport hubs 
TABLE 5: Results of the negative binomial regression model for subway-extending taxi trips.

\begin{tabular}{lccc}
\hline & & Model 3: subway-extending taxi trips \\
Parameter & Coefficient & Standard error & p-value \\
\hline Intercept & $4.58^{* *}$ & 0.258 & 0.000 \\
Subway ridership & $3.14 \mathrm{e}-06^{*}$ & $1.33 \mathrm{e}-06$ & 0.018 \\
Scenic spots & $-6.19 \mathrm{e}-03^{* *}$ & $2.34 \mathrm{e}-03$ & 0.008 \\
Transport hubs & $6.42 \mathrm{e}-03^{* *}$ & $1.56 \mathrm{e}-03$ & 0.000 \\
\hline
\end{tabular}

Note. $*: p<0.01, *: p<0.05,+: p<0.10$.

variables have positive coefficients, and scenic spots variable has a negative coefficient.

\section{Discussion and Conclusions}

Taxi service has been an important component of public transportation for a long time, which is the most flexible mode of public transportation as it can provide door-todoor services. The subway system has developed rapidly and provides fast mass transit services in Beijing. As a result, there exist the competition and corporation between taxi and subway at the same time.

This study investigated the relationship between taxi and subway, with the taxi GPS data, subway ridership data, locations of subway stations, and POIs data. Based on the relationship between taxi and subway, the taxi trips are categorized into three types, subway-competing, subwayextending, and subway-complementing ones. The results showed that the subway-complementing taxi trips take the majority of all trips, which indicates that many trips cannot be satisfied by the subway system alone and the subway network requires further enhancement. In detail, the average travel distances of subway-extending taxi trips are apparently longer than the other two types, which is different from the finding of Wang and Ross [23]. Besides, the average speed of subwayextending taxi trip is significantly higher than the other types. As for spatial distributions, the subway-competing taxi trips are serving the urban area with high subway availability while the subway-extending taxi trips are more likely to serve the peripheral areas. There are different functions of taxi trips and the demand for each function would change with the development of the subway system.

Subway has received rapid development for its sustainability. The efficiency and attractiveness of subway system rely not only on the operation of the subway network but also on the performance of access modes. As an access mode, taxi can increase the access distance for the subway station than walk and bicycle. However, the cost is the major constraint of taxi as an access mode. Fortunately, the emerging of ridesharing helps to decrease the fare as well as the difficulty of hailing a taxi. In addition, the bike-sharing offers a cheap and convenient opportunity to access subway stations, which makes subway more competitive when compared with private cars [41].

The spatial distributions of origin and destination are significantly correlated for both subway and taxi trips. However, the two modes show great differences in spatial distribution. According to the supply and demand of taxis within the buffer zones of subway stations, it was found that there are over-demand of taxis in the urban area and over-supply in the peripheries. Another interesting finding is that both the subway and the taxi have more arrival trips than departure trips in the regions of railway stations and airports.

The results of different negative binomial regression models for different situations indicate that subway ridership plays an important role in explaining differences in taxi trips. In addition, there are several variables positively associated with taxi trips in model 1 and model 2 , including recreation facilities, government agencies, and transport hubs. However, automobile services and life facilities have significantly negative correlations with taxi trips. In model 1 and model 2 , most of independent variables are similar and they have particular variables, respectively. Accommodations variable has a positive correlation with subway-competing taxi trips in model 1, while companies variable has a negative correlation. In model 2 , medical facilities variable is positively correlated with taxi trips in buffer zones of subway stations.

Understanding the relationship between taxi and subway can help to integrate different modes to satisfy passengers' travel demand better. In the future works, comparison between the travel times by taxi and subway with the same origin and destination will be made to identify their respective advantaged travels. However, there are some limitations in this study. The sociodemographic characteristics of passengers are not taken into consideration, which might affect travel demands. The classification method lacks validation, and the distance thresholds utilized in this study require further calibration with more actual data.

\section{Conflicts of Interest}

The authors declare that there are no conflicts of interest regarding the publication of this paper.

\section{Acknowledgments}

The authors would like to acknowledge the financial support for this study provided by the National Natural Science Foundation of China (nos. 71621001, 91746201) and Fundamental Research Funds for Central Universities (no. 2017YJS112).

\section{References}

[1] X. Liang, X. Zheng, W. Lv, T. Zhu, and K. Xu, "The scaling of human mobility by taxis is exponential," Physica A: Statistical 
Mechanics and its Applications, vol. 391, no. 5, pp. 2135-2144, 2012.

[2] W. Wang, L. Pan, N. Yuan, S. Zhang, and D. Liu, "A comparative analysis of intra-city human mobility by taxi," Physica A: Statistical Mechanics and its Applications, vol. 420, pp. 134-147, 2015.

[3] Z. Christoforou, C. Milioti, P. Dionysia, and M. G. Karlaftis, "Investigation of taxi travel time characteristics," in Proceedings of the Transportation Research Board 90th Annual Meeting, 2011.

[4] S. Phithakkitnukoon, M. Veloso, C. Bento, A. Biderman, and C. Ratti, "Taxi-aware map: identifying and predicting vacant taxis in the city," in Proceedings of the International Joint Conference on Ambient Intelligence, pp. 86-95, 2010.

[5] H.-W. Chang, Y.-C. Tai, and Y.-J. J. Hsu, "Context-aware taxi demand hotspots prediction," International Journal of Business Intelligence and Data Mining, vol. 5, no. 1, pp. 3-18, 2010.

[6] C. Kamga, M. A. Yazici, and A. Singhal, "Hailing in the rain: Temporal and weather-related variations in taxi ridership and taxi demand-supply equilibrium," in Proceedings of the Transportation Research Board Annual Meeting, 2013.

[7] J.-J. Lin and T.-Y. Shin, "Does transit-oriented development affect metro ridership?: Evidence from Taipei, Taiwan," Transportation Research Record Journal of the Transportation Research Board, vol. 2063, no. 2063, pp. 149-158, 2008.

[8] J. Choi, Y. J. Lee, T. Kim, and K. Sohn, "An analysis of Metro ridership at the station-to-station level in Seoul," Transportation, vol. 39, no. 3, pp. 705-722, 2012.

[9] C. Roth, S. M. Kang, M. Batty, and M. Barthélemy, "Structure of urban movements: polycentric activity and entangled hierarchical flows," PLoS ONE, vol. 6, no. 1, Article ID e15923, 2011.

[10] Q. Xu, B. Mao, and Y. Bai, "Network structure of subway passenger flows," Journal of Statistical Mechanics Theory \& Experiment, vol. 2016, no. 3, Article ID 033404, 2016.

[11] S. Jiang, W. Guan, W. Zhang, X. Chen, and L. Yang, "Human mobility in space from three modes of public transportation," Physica A Statistical Mechanics Its Applications, vol. 483, 2017.

[12] J. Zhao, W. Deng, Y. Song, and Y. Zhu, "What influences Metro station ridership in China? Insights from Nanjing," Cities, vol. 35, pp. 114-124, 2013.

[13] J. Li, X. Li, D. Chen, and L. Godding, "Assessment of metro ridership fluctuation caused by weather conditions in Asian context: Using archived weather and ridership data in Nanjing," Journal of Transport Geography, vol. 66, 2018.

[14] J. Zhao, W. Deng, Y. Song, and Y. Zhu, "Analysis of Metro ridership at station level and station-to-station level in Nanjing: An approach based on direct demand models," Transportation, vol. 41, no. 1, pp. 133-155, 2014.

[15] M.-J. Jun, K. Choi, J.-E. Jeong, K.-H. Kwon, and H.-J. Kim, "Land use characteristics of subway catchment areas and their influence on subway ridership in Seoul," Journal of Transport Geography, vol. 48, pp. 30-40, 2015.

[16] S. Chandra, M. E. Bari, P. C. Devarasetty, and S. Vadali, "Accessibility evaluations of feeder transit services," Transportation Research Part A: Policy and Practice, vol. 52, pp. 47-63, 2013.

[17] G. Sun, J. Zacharias, B. Ma, and N. M. Oreskovic, "How do metro stations integrate with walking environments? Results from walking access within three types of built environment in Beijing," Cities, vol. 56, pp. 91-98, 2016.

[18] Y.-H. Cheng and Y.-C. Lin, "Expanding the effect of metro station service coverage by incorporating a public bicycle sharing system," International Journal of Sustainable Transportation, pp. $1-12,2018$.
[19] J. Xiong, Z. He, W. Guan, and B. Ran, "Optimal timetable development for community shuttle network with metro stations," Transportation Research Part C: Emerging Technologies, vol. 60, pp. 540-565, 2015.

[20] X. Huang, X. Cao, J. Yin, and X. Cao, "Effects of metro transit on the ownership of mobility instruments in Xian, China," Transportation Research Part D: Transport and Environment, vol. 52, pp. 495-505, 2017.

[21] X. Huang, X. Cao, J. Yin, and X. Cao, "Can metro transit reduce driving? Evidence from Xian, China,” Transport Policy, 2018.

[22] H. H. Hochmair, "Spatiotemporal Pattern Analysis of Taxi Trips in New York City," Transportation Research Record, vol. 2542, pp. 45-56, 2016.

[23] F. Wang and C. L. Ross, "New potential for multimodal connection: exploring the relationship between taxi and transit in New York City (NYC)," Transportation, pp. 1-22, 2017.

[24] X. Qian and S. V. Ukkusuri, "Spatial variation of the urban taxi ridership using GPS data," Applied Geography, vol. 59, pp. 31-42, 2015.

[25] L. Kattan, A. De Barros, and S. C. Wirasinghe, "Analysis of work trips made by taxi in Canadian cities," Journal of Advanced Transportation, vol. 44, no. 1, pp. 11-18, 2010.

[26] Y.-C. Chang, "Factors affecting airport access mode choice for elderly air passengers," Transportation Research Part E: Logistics and Transportation Review, vol. 57, pp. 105-112, 2013.

[27] G. Akar, "Ground access to airports, case study: Port Columbus International Airport," Journal of Air Transport Management, vol. 30, pp. 25-31, 2013.

[28] I. Gokasar and G. Gunay, "Mode choice behavior modeling of ground access to airports: A case study in Istanbul, Turkey," Journal of Air Transport Management, vol. 59, pp. 1-7, 2017.

[29] Z. Ma, M. Urbanek, M. A. Pardo, J. Y. Chow, and X. Lai, "Spatial welfare effects of shared taxi operating policies for first mile airport access," International Journal of Transportation Science and Technology, vol. 6, no. 4, pp. 301-315, 2017.

[30] M. Anil Yazici, C. Kamga, and A. Singhal, "Modeling taxi drivers' decisions for improving airport ground access: John F. Kennedy airport case," Transportation Research Part A: Policy and Practice, vol. 91, pp. 48-60, 2016.

[31] M. L. Tam, M. L. Tam, and W. H. K. Lam, "Analysis of airport access mode choice: a case study in Hong Kong," Journal of the Eastern Asia Society for Transportation Studies, vol. 6, pp. 708723, 2005.

[32] M. Givoni and P. Rietveld, "The access journey to the railway station and its role in passengers' satisfaction with rail travel," Transport Policy, vol. 14, no. 5, pp. 357-365, 2007.

[33] BJTRC, Beijing Transport Annual Report (2015), 2017, http://www.bjtrc.org.cn/.

[34] Z. He, L. Zheng, P. Chen, and W. Guan, "Mapping to cells: A simple method to extract traffic dynamics from probe vehicle data," Computer-Aided Civil and Infrastructure Engineering, vol. 32, no. 3, pp. 252-267, 2017.

[35] Z. He and L. Zheng, "Visualizing Traffic Dynamics Based on Floating Car Data," Journal of Transportation Engineering, Part A: Systems, vol. 143, no. 5, 2017.

[36] Amap, The coordinates picker system in Amap, 2017, http://lbs .amap.com/console/show/picker.

[37] S. Beijing, The adjacent station distance of Beijing Subway, 2017, http://www.bjsubway.com/station/zjgls/.

[38] D. B. Hess, "Access to public transit and its influence on ridership for older adults in two U.S. cities," Journal of Transport and Land Use, vol. 2, no. 1, pp. 3-27, 2009. 
[39] A. M. El-Geneidy, P. R. Tétreault, and J. Surprenant-Legault, "Pedestrian access to transit: Identifying redundancies and gaps using a variable service area analysis," in Proceedings of the Transportation Research Board Meeting, 2010.

[40] H. Eftekhar, L. Creemers, and M. Cools, "Effect of Traveler's Nationality on Daily Travel Time Expenditure Using ZeroInflated Negative Binomial Regression Models: Results from Belgian National Household travel Survey," Transportation Research Record, vol. 2565, pp. 65-77, 2016.

[41] S. Jiang, W. Guan, Z. Wang, and 1. Yang, "Improving the accessibility of metro system by using bike-metro integration," Working paper, 2018. 


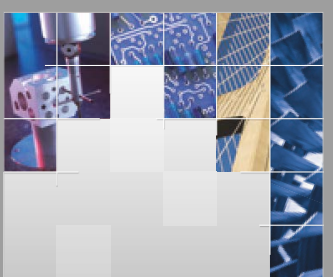

\section{Enfincering}
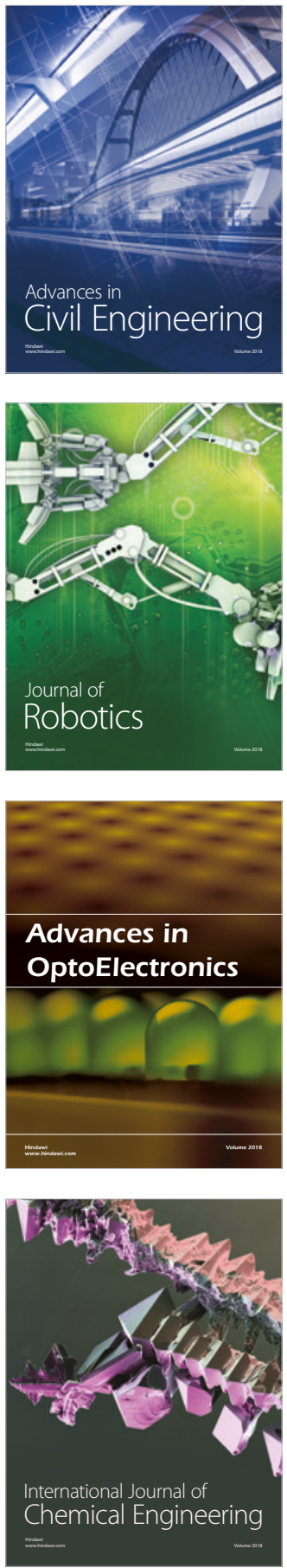

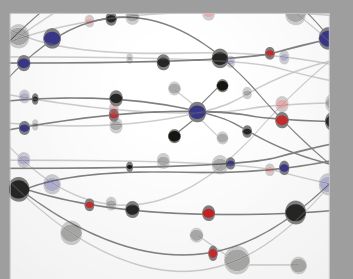

\section{Rotating \\ Machinery}

The Scientific World Journal

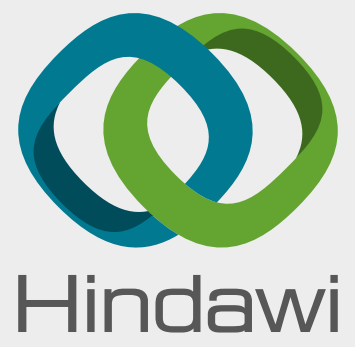

Submit your manuscripts at

www.hindawi.com
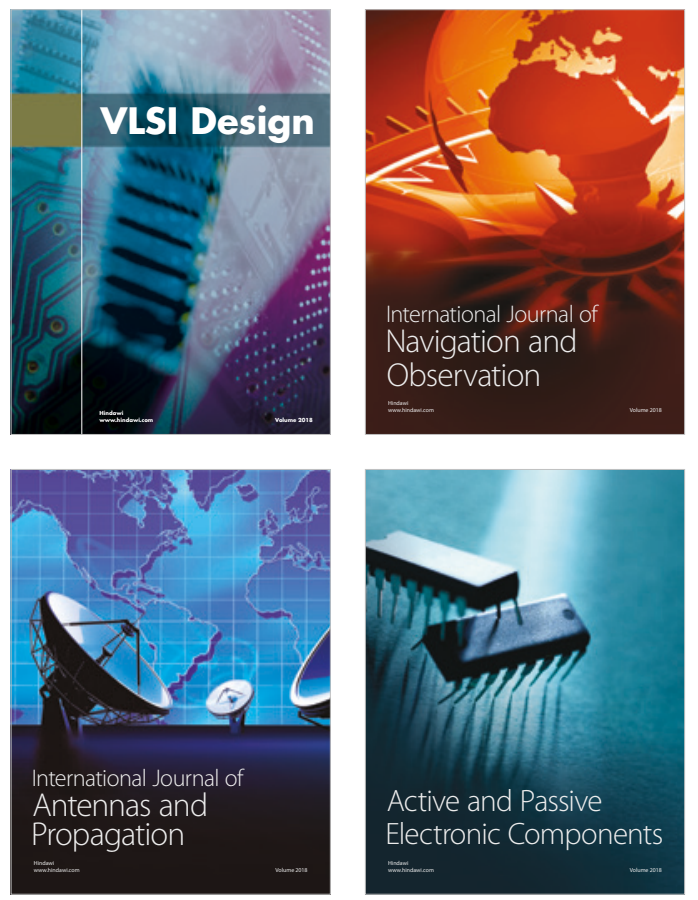
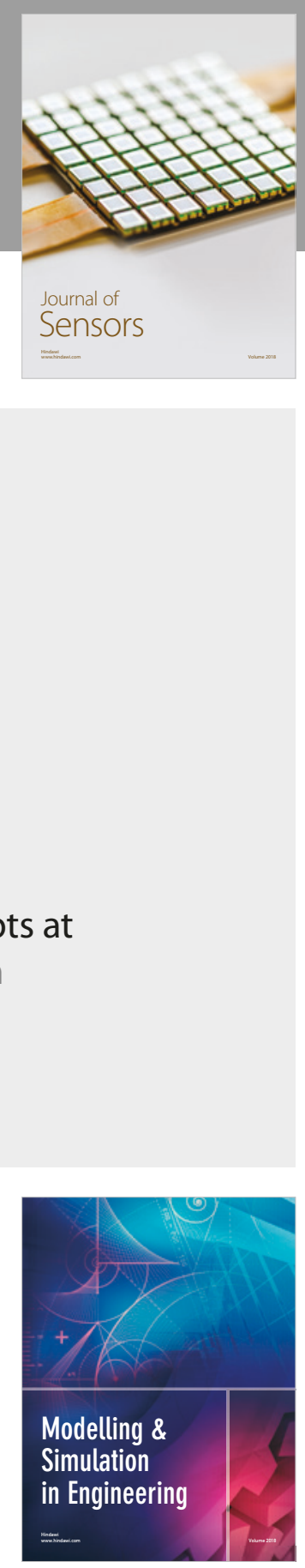

\section{Advances \\ Multimedia}
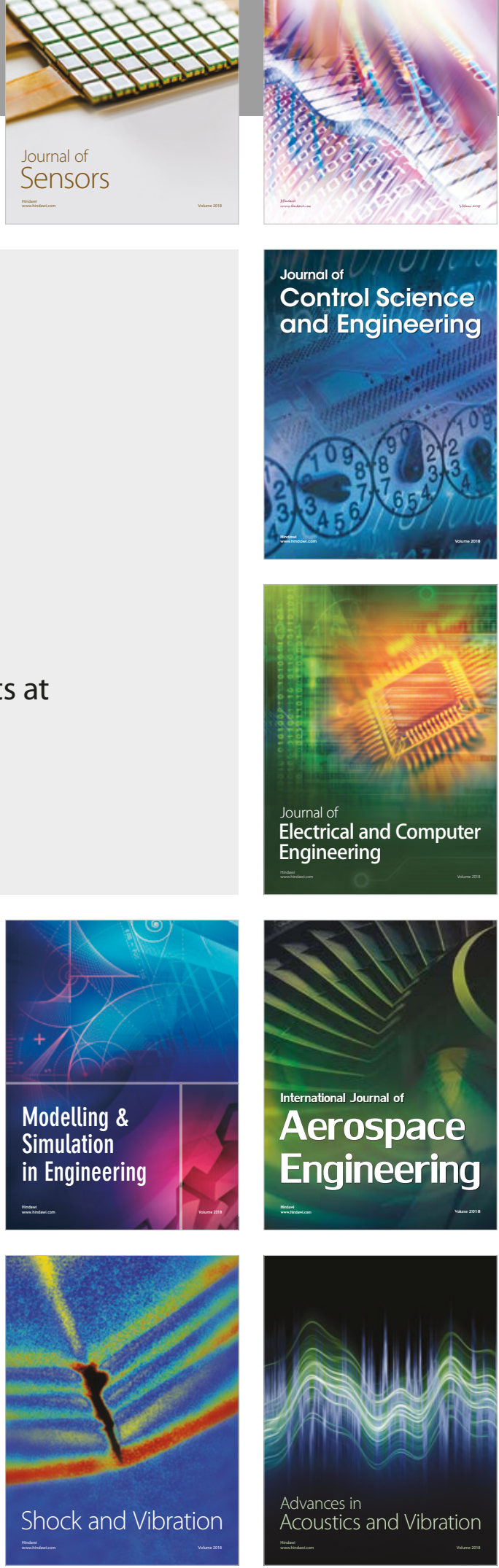